\title{
A Rare Case Report of Ruptured Cyst Presenting With Acute Meningitis in an Untreated Neurocysticercosis \\ Hammam Shereef ${ }^{1}$, Mahad Ahmed ${ }^{1}$, Mohamedanwar Ghandour ${ }^{2}$, Gino Tapia-Zegarra ${ }^{3}$
}

1. Internal Medicine, Beaumont Health, Dearborn, USA 2. Internal Medicine/Nephrology, Wayne State University Detroit Medical Center, Detroit, USA 3. Infectious Diseases Consultants, San Antonio, USA

Corresponding author: Hammam Shereef, hammamhakim@gmail.com

\begin{abstract}
Neurocysticercosis (NCC) rarely presents as acute meningitis; however, when it does, it is not distinguishable clinically from other more common infectious etiologies. Here, we report a case of NCC presenting as acute meningitis, which also highlights the importance of brain MRI imaging rather than CT where possible, the need to include MRI of the spine in patients with the subarachnoid disease, and the limitations of NCC antigen detection assay in cerebrospinal fluid when used in ventriculoperitoneal shunt specimens. A prolonged course of albendazole, praziquantel, and corticosteroids led to the resolution of our patient's NCC.
\end{abstract}

Categories: Infectious Disease
Keywords: neurocysticercosis, meningitis, subarachnoid

\section{Introduction}

Neurocysticercosis (NCC) is the most common parasitic central nervous system (CNS) infection in human beings [1]. It follows the ingestion of the larval stage (Cysticercus cellulosae) of the swine tapeworm Taenia solium. NCC is the most common cause of secondary seizure disorder for which patients may need to be on long-term anti-epileptic medication [2]. Approximately 50 million persons worldwide are estimated to have cysticercosis infection, and as per WHO, around 50 thousand die annually because of NCC and seizures related to NCC.

Cysticercosis is endemic in many regions of Central and South America, sub-Saharan Africa, India, and Asia. A review of the national database found 18,584 hospitalizations of NCC in the USA between 2003 and 2012. Among them, 74\% were Hispanics [3].

NCC can present as a parenchymal infection (ParNCC), which is the most common form and carries a better prognosis than the less common extraparenchymal disease (ExPNCC). Seizures are the main presenting feature of ParNCC in up to 80\%, whereas ExPNCC can manifest as an intraventricular form followed by the subarachnoid, spinal, and ocular NCC [4].

\section{Case Presentation}

We report the case of a 31-year-old male from rural Mexico who immigrated to the USA in 2001 with no prior medical and surgical histories; he initially presented to our hospital in 2009 with obtundation and no report of seizures, fever, or other complaints. The patient worked in farms in rural Mexico, where he had eaten undercooked pork. Workup at that time revealed hydrocephalus with multiple calcified brain lesions on brain CT with no cysts or edema (Figure 1) with positive serum T. solium serology and negative blood QuantiFERON® (Qiagen, Hilden, Germany). The patient was treated with a ventriculoperitoneal (VP) shunt placement. CT of the brain lesions did not show active cysticercosis; thus, no anti-parasitic treatment was given at initial presentation. 


\section{Cureus}

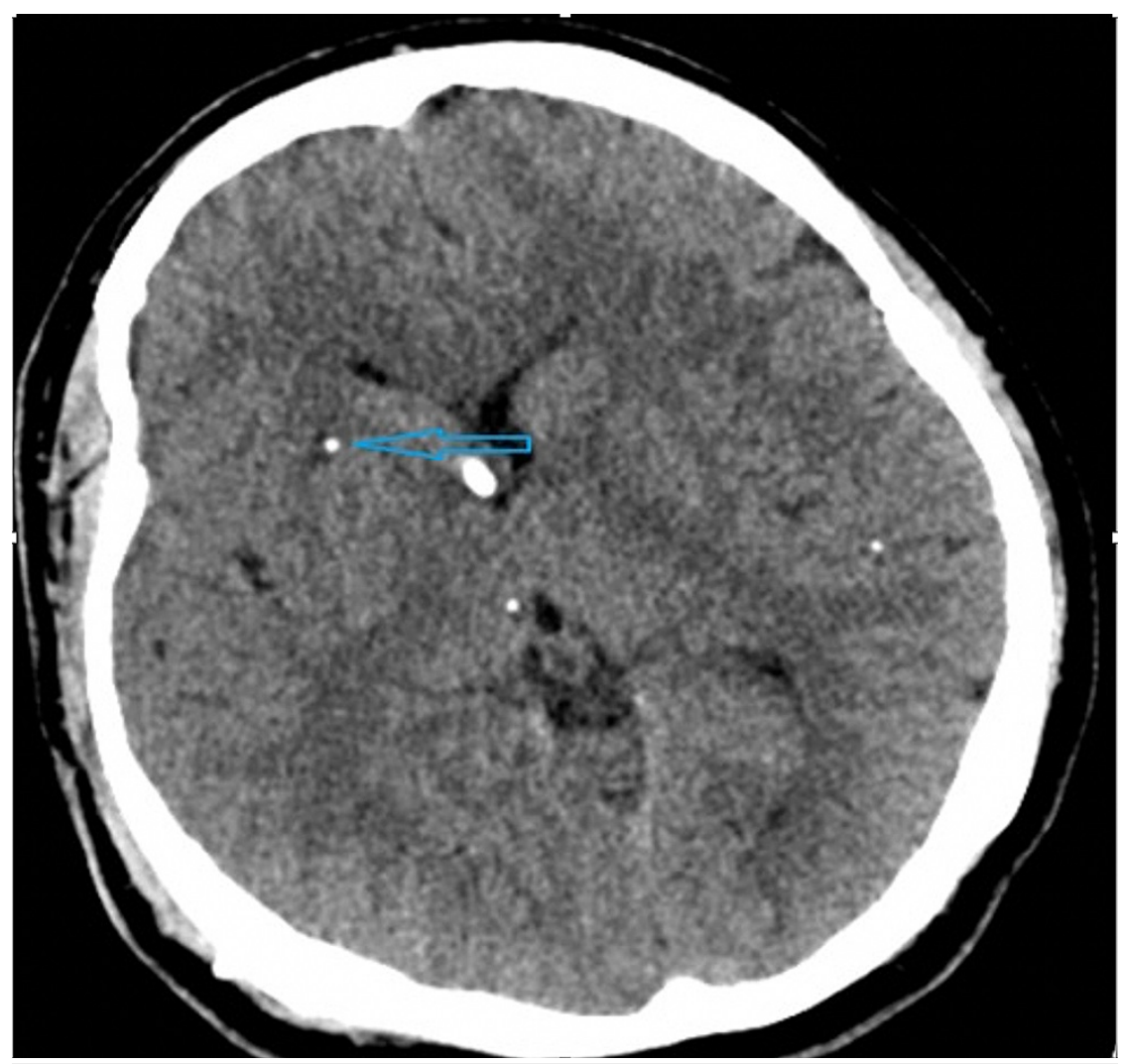

FIGURE 1: CT of the head showing calcifications (blue arrow).

In July 2017, the patient presented with chief complaints of headache and fever of one-day duration but no other symptoms. Physical examination was unremarkable, including no meningeal signs or focal neurologic deficit and unremarkable funduscopic examination. Non-contrast CT of the head showed stable calcified brain lesions since 2009 and no cysts/edema or new changes. Lumbar puncture (LP) revealed cerebrospinal fluid (CSF) white blood cell (WBC) count of 3,320/cumm (81\% neutrophils, $11 \%$ lymphocytes, no eosinophils), red blood cell (RBC) of 80/cumm, glucose of $9 \mathrm{mg} / \mathrm{dL}$ (glucose in blood of $198 \mathrm{mg} / \mathrm{dL}$ ) protein of $687 \mathrm{~g} / \mathrm{dL}$ (serum protein of 6.4; normal range: $6.0-8.0 \mathrm{~g} / \mathrm{dL}$ ). On the same day of LP, VP shunt CSF analysis revealed WBC of 20 (34\% neutrophils, 59\% lymphocytes, no eosinophils), RBC of 16,900 (traumatic tap reported), glucose of $124 \mathrm{mg} / \mathrm{dL}$, and protein of $343 \mathrm{~g} / \mathrm{d}$. CSF from VP shunt grew Propionibacterium acnes. CSF from LP blood cultures did not reveal any organisms, and CSF polymerase chain reactions (PCRs) were negative for herpes simplex, varicella-zoster, cytomegalovirus, adenovirus, and enterovirus. CSF NCC antigen in the blood (done at the CDC) and CSF (from VP shunt) were negative; however, EITB (enzymelinked immunoelectrotransfer blot) NCC antibody was positive in CSF and serum.

The patient completed two weeks of empiric intravenous ceftriaxone and vancomycin and became asymptomatic within two days of his hospitalization. Pertinent findings from cervical, thoracic, lumbar, and brain contrast MRIs (Figures 2,3) showed peripherally enhancing cystic lesion measuring $1 \mathrm{~cm}$ with illdefined mural nodule measuring $5 \mathrm{~mm}$ identified along the dorsal surface of upper cervical cord at the level of the foramen magnum. Local leptomeningeal enhancement was present along the dorsal surface of the upper cervical cord extending inferiorly to the $\mathrm{C} 1$ level and superiorly into the foramen of Magendie. The small extra-axial fluid collection extends posteriorly into the inferior left posterior fossa along the inferiormedial margin of the left cerebellar hemisphere, directly posterior to the left cerebellar tonsil measuring $13 \mathrm{x}$ $6 \mathrm{~mm}$, without marginal enhancement, which felt to be reactive to ruptured cyst inflammation. These findings were compatible with NCC cyst rupture. 


\section{Cureus}

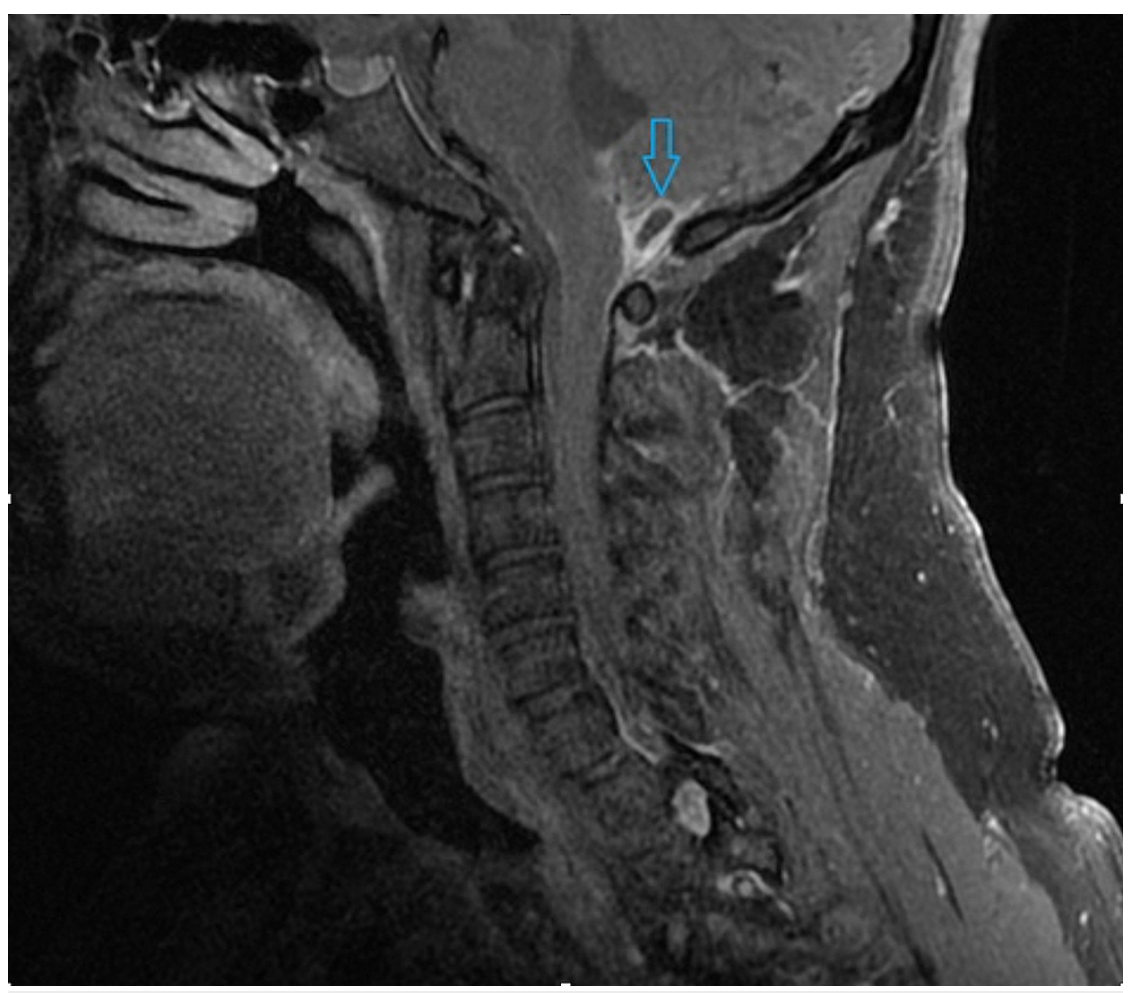

FIGURE 2: T1-weighted image with contrast showing leptomeningeal enhancement dorsal surface of the upper cervical cord (blue arrow).

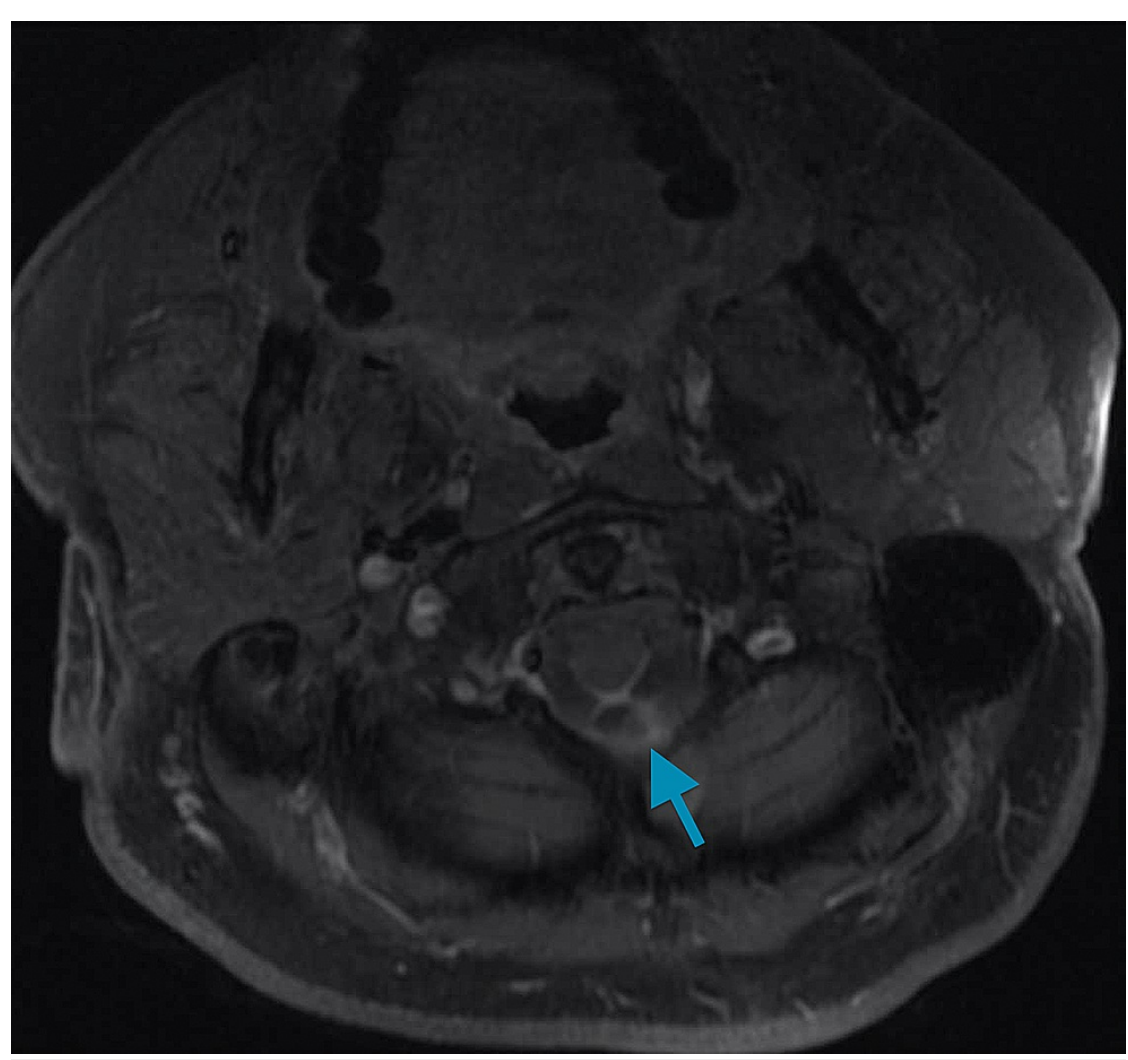

FIGURE 3: Enhancement dorsal to the cord at the level of the foramen magnum (blue arrow).

\section{Follow-up}


The patient completed six months of praziquantel $1,800 \mathrm{mg}$ three times daily and albendazole $400 \mathrm{mg}$ twice daily, along with long-term prednisone slowly tapered with good tolerability and resolution of the enhancing cystic lesion along the upper cervical cord at the level of the foramen magnum, leptomeningeal enhancement, and inflammatory signs per follow-up brain MRI performed in October 2017. These MRI findings remained unchanged without inflammatory changes on repeat brain MRI performed in January 2018 upon discontinuation of anti-parasitic therapy, and the patient remains asymptomatic to date.

\section{Discussion}

It is estimated that the NCC incidence in the United States range from 0.2 to 0.6 cases per 100,000 general population and 1.5-5.8 cases per 100,000 Hispanics [5]. ExPNCC (subarachnoid, meningeal, and intraventricular space) is less common than parenchymal infection (ParNCC). The manifestations of ExPNCC range from asymptomatic lesions to meningitis, stroke, and hydrocephalus [6,7]. Subarachnoid NCC can present with communicating hydrocephalus, meningitis, stroke, or focal neurologic deficits [7]. A study published in 2004 reported that $91 \%$ of NCC cases presented with parenchymal infection, subarachnoid cysts in $2 \%$, ventricular cysts in $6 \%$, and hydrocephalus in $16 \%$ of NCC cases. [8]. In contrast, a case series between (1997-2005) from Houston, Texas, United States, included a total of 111 patients, of whom 60 patients (54\%) of had parenchymal disease, 22 patients (20\%) with intraventricular involvement, 13 patients (12\%) had subarachnoid disease, and 13 (12\%) had calcifications only; moreover, two patients only had hydrocephalus, and one patient developed ocular cysticercosis [9]. Moreover, a case series from New Mexico reported that $30 \%$ of cases were of ExPNCC [10]. Cysticercal meningitis (CM), which represents the host inflammatory response to the parasite, is usually chronic and occurs in less than $8 \%$ of adult NCC cases $[6,8]$. There have been few cases of NCC presenting as acute meningitis [8,11], although it is probably an underreported presentation. It is unclear if any reported cases were due to a ruptured NCC cyst, which was present in our patient.

The recommended EITB assay (the most reliable test for the detection of antibodies specific for T. solium antigens in serum or CSF) has a specificity approaching $100 \%$ and a sensitivity varying from $70 \%$ to $90 \%$ depending on the number of cysts present $[12,13]$. However, A positive cysticercosis antibody does not distinguish between previous or active infection. Besides, in endemic regions, there is a positive background seroprevalence of 5-20\% [14]. Therefore, parasite antigen detection assays seem useful for both diagnosis (which are very specific for viable infection and its detection in CSF is specific to CNS infection) and treatment monitoring (parasite antigens typically fall after successful treatment). Antigen assays are more sensitive when done in CSF, which, in turn, can be falsely negative mainly due to a single intracranial cysticercus $[13,15]$. Standardized antigen detection assays are not commercially available in the United States currently [7].

Treatment of subarachnoid NCC may include prolonged courses of albendazole or combinations of praziquantel and albendazole until radiological resolution of viable cysticerci in conjunction with corticosteroids use to downregulate the immune response associated with anti-parasitic use [7,16,17].

\section{Conclusions}

There is a clinical dilemma when a patient develops acute meningitis in the setting of subarachnoid NCC and the presence of a VP shunt device, which is always concerning for device infection. In terms of diagnostic criteria, our patient would have been diagnosed with device infection (by P. acnes) by which he would have warranted device removal as recommended by the Infectious Diseases Society of America (IDSA). However, based on our patient's radiologic and serologic diagnostic criteria met for NCC as suggested by IDSA, quick improvement without device removal made the diagnosis of shunt infection improbable, leading to the diagnosis of a ruptured NCC cyst as the only pathologic process in our patient and P. acnes as being a CSF specimen contaminant and therefore avoiding shunt removal. All patients with hydrocephalus and risk factors for NCC should have a thorough evaluation for NCC including brain MRI, if possible, to look for additional cysticerci as those cases can also have asymptomatic involvement with additional lesions in the parenchyma, subarachnoid space, or ventricles with important prognostic and monitoring implications. Given its strong association with spinal involvement, all patients with intracranial subarachnoid disease should also undergo an MRI of the spine. If the NCC antigen detection assay is used in a patient with a VP shunt, it is best to obtain the CSF specimen from an LP, particularly in subarachnoid NCC, where circulating antigens are almost always present.

\section{Additional Information \\ Disclosures}

Human subjects: Consent was obtained or waived by all participants in this study. Conflicts of interest: In compliance with the ICMJE uniform disclosure form, all authors declare the following: Payment/services info: All authors have declared that no financial support was received from any organization for the submitted work. Financial relationships: All authors have declared that they have no financial relationships at present or within the previous three years with any organizations that might have an interest in the submitted work. Other relationships: All authors have declared that there are no other relationships or activities that could appear to have influenced the submitted work.

\section{References}

1. Garcia HH, Gonzalez AE, Gilman RH: Diagnosis, treatment and control of Taenia solium cysticercosis. Curr Opin Infect Dis. 2003, 16:411-419. 10.1097/00001432-200310000-00007

2. Del Brutto OH, Santibanez R, Noboa CA, Aguirre R, Diaz E, Alarcon TA: Epilepsy due to neurocysticercosis 


\section{Cureus}

analysis of 203 patients. Neurology. 1992, 42:389-389. 10.1212/wnl.42.2.389

3. O'Neal SE, Flecker RH: Hospitalization frequency and charges for neurocysticercosis, United States, 20032012. Emerg Infect Dis. 2015, 21:969. 10.3201/eid2106.141324

4. Marcin Sierra M, Arroyo M, Cadena Torres, et al.: Extraparenchymal neurocysticercosis: demographic, clinicoradiological, and inflammatory features. Plos Negl Trop Dis. 2017, 11:0005646. 10.1371/journal.pntd.0005646

5. Serpa JA, White AC: Neurocysticercosis in the United States. Pathog Glob Health. 2012, 106:256-260. 10.1179/2047773212Y.0000000028

6. Kelesidis T, Tsiodras S: Extraparenchymal neurocysticercosis in the United States. Am J Med Sci. 2012, 1:7982. 10.1097/MAJ.0b013e31823e6565

7. White AC Jr, Coyle CM, Rajshekhar V, et al.: Diagnosis and treatment of neurocysticercosis: 2017 Clinical Practice Guidelines by the Infectious Diseases Society of America (IDSA) and the American Society of Tropical Medicine and Hygiene (ASTMH). Clin Infect Dis. 2018, 66:1159-1163. 10.1093/cid/ciy157

8. Wallin MT, Kurtzke JF: Neurocysticercosis in the United States: review of an important emerging infection . Neurology. 2004, 9:1559-1564. 10.1212/01.wnl.0000142979.98182.ff

9. Serpa JA, Graviss EA, Kass JS, White AC Jr: Neurocysticercosis in Houston, Texas: an update . Medicine. 2011, 90:81-86. 10.1097/MD.0b013e318206d13e

10. Figueroa JJ, Davis LE, Magalhaes A: Extraparenchymal neurocysticercosis in Albuquerque, New Mexico. J Neuroimaging. 2011, 21:38-43. 10.1111/j.1552-6569.2009.00452.x

11. Earnest MP, Reller LB, Filley CM, Grek AJ: Neurocysticercosis in the United States: 3S cases and a review . Rev Infect Dis. 1987, 5:961-979. 10.1093/clinids/9.5.96

12. Garcia HH, Nash TE, Del Brutto OH: Clinical symptoms, diagnosis, and treatment of neurocysticercosis . Lancet Neurol. 2014, 13:1202-1215. 10.1016/S1474-4422(14)70094-8

13. Landscape analysis: management of neurocysticercosis with an emphasis on low- and middle-income countries. (2015). Accessed: November 1, 2020:

https://apps.who.int/iris/bitstream/handle/10665/152896/WHO_HTM_NTD_NZD_2015.05_eng.pdf;jsessionid=54C8A54AE472FD(

14. Del Brutto OH, Nash TE, White AC Jr, et al.: Revised diagnostic criteria for neurocysticercosis. J Neurol Sci. 2017 Jan, 15:202-210. 10.1016/j.jns.2016.11.045

15. Gabriel S, Blocher J, Dorny P, et al.: Added value of antigen ELISA in the diagnosis of neurocysticercosis in resource poor settings. PLoS Negl Trop Dis. 2012, 6:1851. 10.1371/journal.pntd.0001851

16. Tunkel AR, Hasbun R, Bhimraj A, et al.: 2017 Infectious Diseases Society of America's Clinical Practice Guidelines for healthcare-associated ventriculitis and meningitis. Clin Infect Dis. 2017, 64:34-65. 10.1093/cid/ciw861

17. Callacondo D, Garcia HH, Gonzales I, Escalante D, Nash TE: High frequency of spinal involvement in patients with basal subarachnoid neurocysticercosis. Neurology. 2012, 78:1394-1400. 10.1212/WNL.0b013e318253d641 